\title{
Experimental Investigation of Self-Assembled Opal Structures by Atomic Force Microscopy, Spectroscopic Ellipsometry and Reflectometry
}

\author{
Natalia Alekseeva ${ }^{1}$, Grigory Cema ${ }^{1}$, Aleksey Lukin ${ }^{1}$, Svetlana \\ Pan'kova $^{1}$, Sergei Romanov ${ }^{2}$, Vladimir Solovyev ${ }^{1}$, \\ Victor Veisman ${ }^{1}$ and Mikhail Yanikov ${ }^{1}$
}

\footnotetext{
${ }^{1}$ Department of Physics, Faculty of Physics and Mathematics, Pskov State University, Lenin Square 2, 180000 Pskov, Russia;

e-mail: kaf-phy@psksu.ru

${ }^{2}$ Institute of Optics, Information and Photonics, University of Erlangen-Nürnberg, Haber Str. 9a, 91058 Erlangen, Germany; Ioffe Physical Technical Institute,

Polytechnicheskaya Street, 26,194021 St. Petersburg, Russia,

e-mail:Sergei.Romanov@mpl.mpg.de
}

\section{Received 19 September 2013, Accepted 15 November 2013,} Published 10 December 2013

\begin{abstract}
Self-assembled opal crystals (bulk silica opals and PMMA thin opal films) have been studied by atomic force microscopy (AFM) and optical spectroscopy. Reflectance and transmittance spectra $(R(\lambda)$ and $T(\lambda)$, respectively) as well as spectra of ellipsometric parameters $\Psi(\lambda)$ and $\Delta(\lambda)$ demonstrate pronounced changes with changing the angle of light incidence. Diameters of spheres obtained from AFM-images correspond to those obtained from Bragg fit to the diffraction resonance dispersions. The band of light losses detected by ellipsometry at the spectral range of avoided band crossing of opal eigenmodes was assigned to the energy exchange between these modes.
\end{abstract}

Keywords: opal, photonic crystal, Bragg diffraction, photonic bandgap structure, reflectance and transmittance spectra, ellipsometry, atomic force microscopy.

Journal of Self-Assembly and Molecular Electronics, Vol. 1, 209-222.

doi: 10.13052/jsame2245-4551.124

(c) 2014 River Publishers. All rights reserved. 


\section{Natalia Alekseeva et.al}

\section{Introduction}

In the last decades opal crystals attracted considerable attention as nanoporous templates for the fabrication of various composite materials. Closely packed silica or poly-methyl methacrylate (PMMA) beads of self-assembled synthetic opal (see the surface image in Fig. 1) form the face centered cubic (FCC) lattice [4]. Since the diameters $D$ of beads are in the range of hundreds nanometers, opals are widely used as 3-dimensional photonic crystals for the visible [1, 2, 3].

Optical properties of 3-dimensional opal crystals are usually characterized by the angle-resolved reflectance and transmittance spectroscopy, whereas their structure is examined by scanning electronic microscopy (SEM) and atomic force microscopy (AFM). Considering the high costs of SEM equipment, the SPM methods in combination with digital processing may become prospective and competitive for express investigation of opal-based materials [5].

Among other optical techniques, ellipsometry is designed to provide such characteristics as the refractive index $n$ and the extinction coefficient $k$ of the material under study. Ellipsometry determines the change in polarization of reflected (or transmitted) light from a sample by measuring two parameters $\Psi$ and $\Delta$ that characterize the relative change in the amplitudes of the $\mathrm{p}$ - and

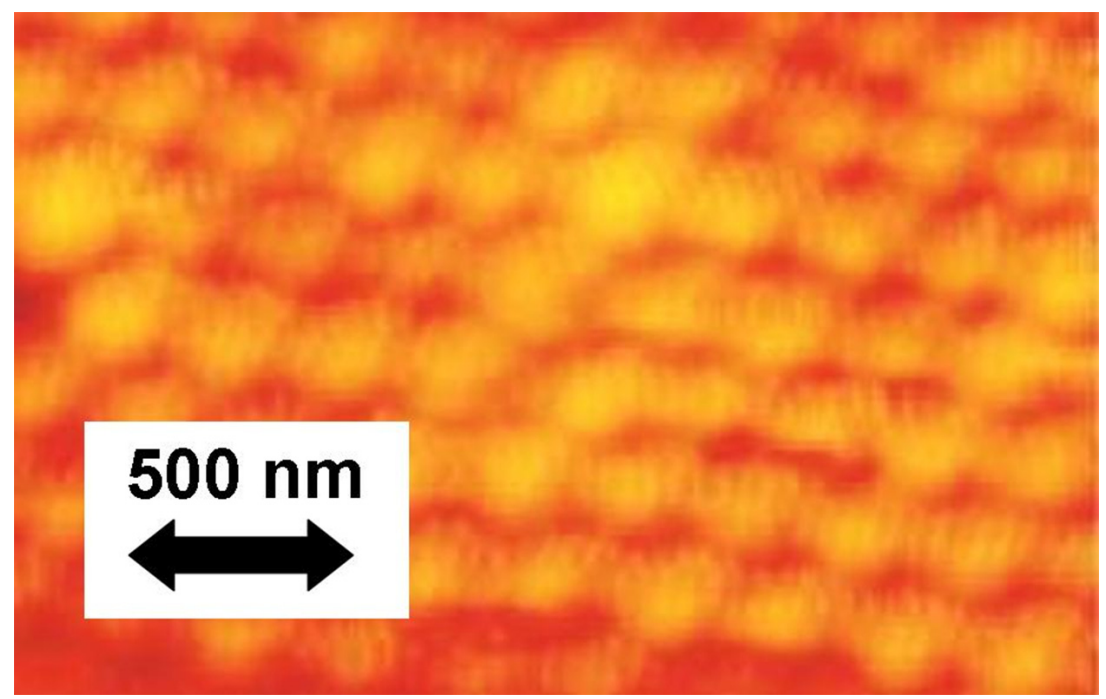

Figure 1 AFM image of the surface of self-organized opal sample 
s-polarized waves and the phase shift between them:

$$
\exp (i \Delta) \tan \Psi=\frac{R_{p}}{R_{s}}
$$

where $R_{p}$ and $R_{s}$ are the reflection coefficients [6]. To our knowledge, only in few cases [7-9] this method was used for characterization of opal structures. In this paper, we compare the spectra of ellipsometric parameters with conventional reflectance spectra for bulk and thin film opal samples.

\section{Experimental Procedures}

Thin film opals with typical sample thickness of about 10 microns were prepared on glass substrates by noise-assisted crystallization from a diluted suspension of PMMA spheres in a vertically moving meniscus [10]. Bulk opal matrices were synthesized by sedimentation of silica beads in Central Research Technology Institute "Technomash" (Moscow) [11]. AFM images were obtained by scanning probe microscope «NanoEducator» (NT-MDT, Moscow).

Angular resolved reflectance and transmittance spectra $(R(\lambda)$ and $T(\lambda)$, respectively) were measured under illumination by unpolarized white light from a tungsten lamp using a collimated beam. Reflected or transmitted light was recorded by USB650 Red Tide spectrometer (Ocean Optics, Inc.). To extract the refractive index and extinction spectra we used the spectroscopic ellipsometer "Ellips-1891" (Novosibirsk), working in the static photometric mode without any rotating elements or modulators [6].

\section{Results \& Discussion}

The shift of the Bragg resonance wavelength $\lambda$ as a function of the angle of light incidence $\theta$ obeys the combined Bragg $(2 a \cos \beta=m \lambda / n$, where $m$ is an integer) and Snell's $(n \sin \beta=\sin \theta)$ laws:

$$
\lambda^{2}=4 a^{2} n^{2}-4 a^{2} \sin ^{2} \theta
$$

where $a=0.816 D$ is the interplane distance for (111) planes of the FCC lattice and $n=n_{\text {eff }}$ represents the effective refractive index of the opal.

One can see the standard "blue" shift of the Bragg resonance band in reflectance spectra $R(\lambda)$ of the bulk opal along the increase of the angle $\theta$. According to our experimental results the similar shift can be observed in 


\section{Natalia Alekseeva et.al}

$\Psi(\lambda)$ spectra (Fig. 2). In the case of better ordered PMMA thin opal films [12, 13], the dispersion of the (111) diffraction resonance deviates from the Bragg law (equation (2)) at incidence angles around $\theta \approx 51^{\circ}$ due to multiple wave diffraction at (111) and (111) or (200) planes. One can clearly observe the avoided crossing of these Bragg resonances in the interval $45^{\circ} \leq \theta \leq 55^{\circ}$ in reflectance and transmittance spectra (Fig. 3), where it appears in the form of double peaks.

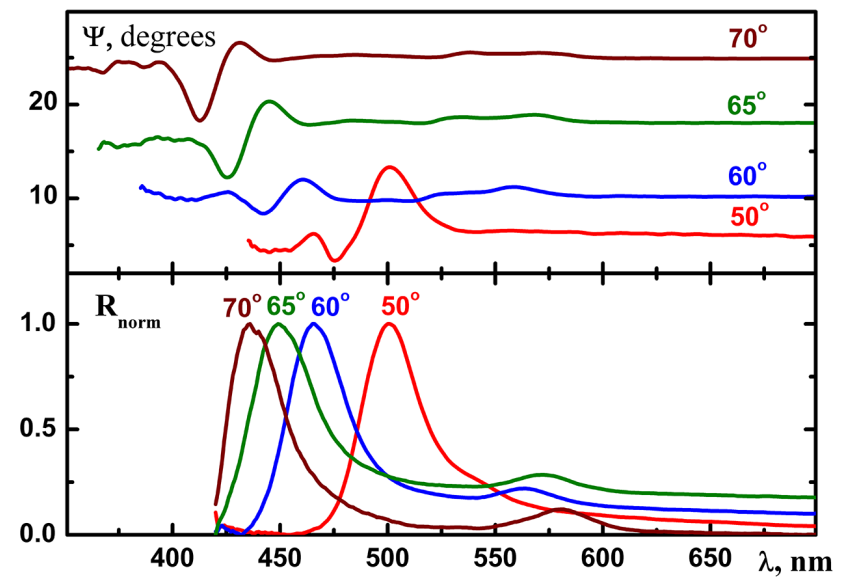

Figure 2 Ellipsometric parameter $\Psi(\lambda)$ and normalized reflectance spectra $R(\lambda)$ of bulk opal at different angles of light incidence

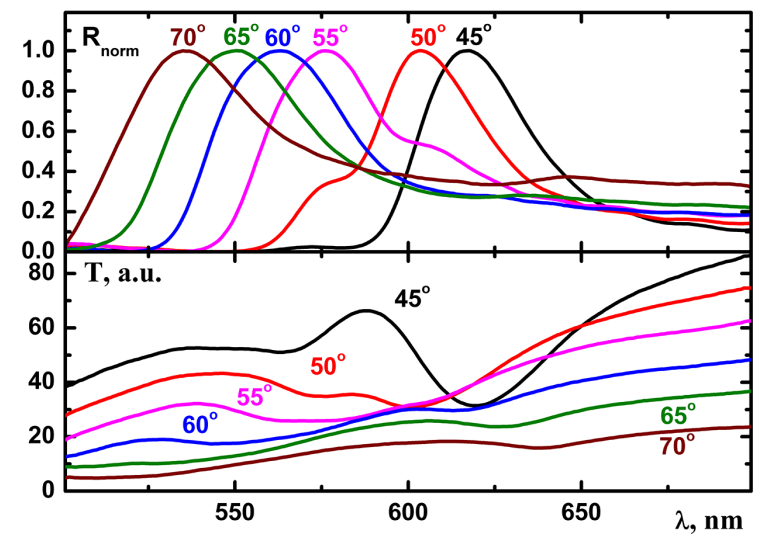

Figure 3 Normalized reflectance and transmittance spectra of the PMMA thin opal film at different angles of light incidence. 
The angle dependence of the (111) Bragg resonance over the broad range of angles is linear in squared coordinates $\lambda^{2}=f\left(\sin ^{2} \theta\right)$ with the Pearson's correlation coefficient $r=0.999$ (Fig. 4). In this case one can use equation (2) to calculate the diameter of opal spheres $D=315 \mathrm{~nm}$. Our previous experiments [14] have shown that the opal sphere diameters obtained from SPM-images and those calculated from Bragg reflectance spectra for opal samples agree with each other within the experimental error. The effective refractive index of $n_{\text {eff }}=1.396$ was extracted from the Bragg fit (2) in the case of the thin opal film.

The value of refractive index $n_{\text {eff }}$ can be also estimated using the effective medium approximation:

$$
n_{\text {eff }}^{2}=f n_{1}^{2}+(1-f) f n_{2}^{2},
$$

where $f=0.26$ is the volume fraction of air voids in the FCC crystal of touching spheres, $n_{1}=n_{\text {air }}, n_{2}=1.489$ is the refractive index of PMMA beads, so we have $n_{\text {eff }}=1.379$ in the good agreement with the index extracted from the Bragg fit.

In the case of the bulk silica opal, the effective index of refraction, as calculated from the dispersion of the (111) Bragg resonance (eq. (2)) (Fig. 4), agrees with the ellipsometric data as well. In our bulk silica opals the porosity

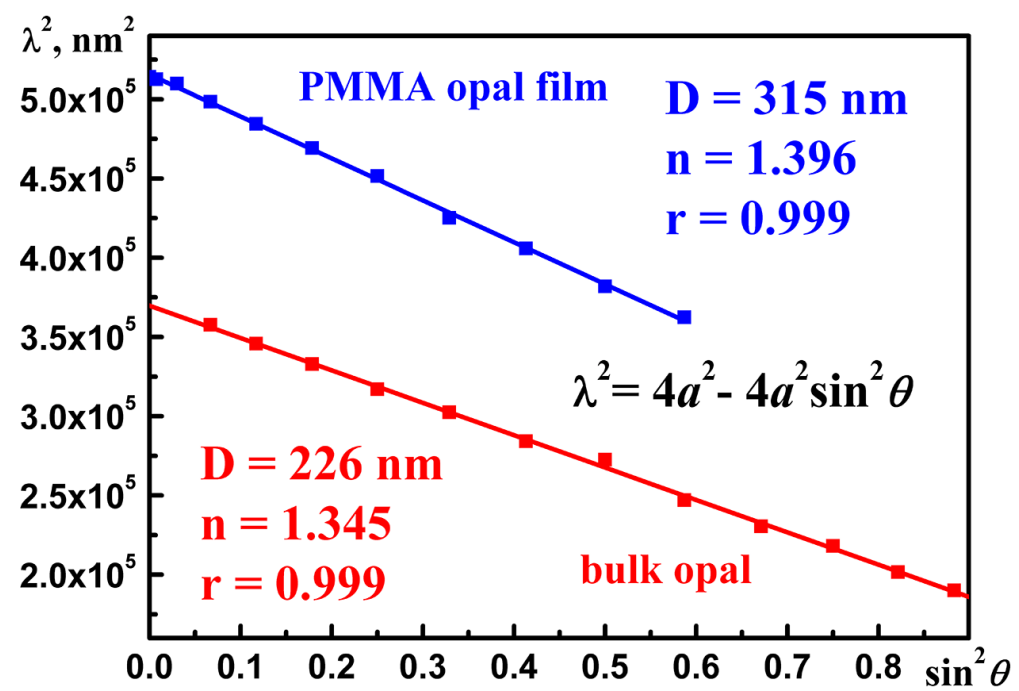

Figure 4 Angular dispersion of the (111) Bragg resonance in the PMMA thin opal film and in the bulk silica opal. 


\section{Natalia Alekseeva et.al}

is $f=0.33$, because the silica beads contain pores in their internal structure. Then one can estimate the same value of the effective refractive index from equation (3): $n_{\text {eff }}=1.356$.

The spectra of the refractive index $n(\lambda)$ and the extinction coefficient $k(\lambda)$ (Fig. 5) of the thin opal film were calculated from the ellipsometric data. To the best of our knowledge, no interpretation of such data can be found in the current literature. We suggest the qualitative model, which takes into account the fact that light in $\mathrm{PhC}$ is transported by the Bloch modes. As per se, the state of linear polarization of the incident light is not preserved in the opal $\mathrm{PhC}$. Calculations show that the E-vector in the Bloch mode can point in different directions for different points of the unit cell. Moreover, the field orientation rapidly changes along changes of the frequency and the quasi-wavevector of the mode [15]. This consideration makes void the standard interpretation of ellipsometric data, which is designed to homogeneous media.

Tentative explanation of $n(\lambda), k(\lambda)$ spectra can be obtained with the help of the energy band structure of the opal $\mathrm{PhC}$ calculated using plane wave expansion method [12]. Fig. 6 shows the fragment of this structure in the range of the avoided band crossing that is relevant to experimental data. In contrast to homogeneous media, in which index of refraction is defined by

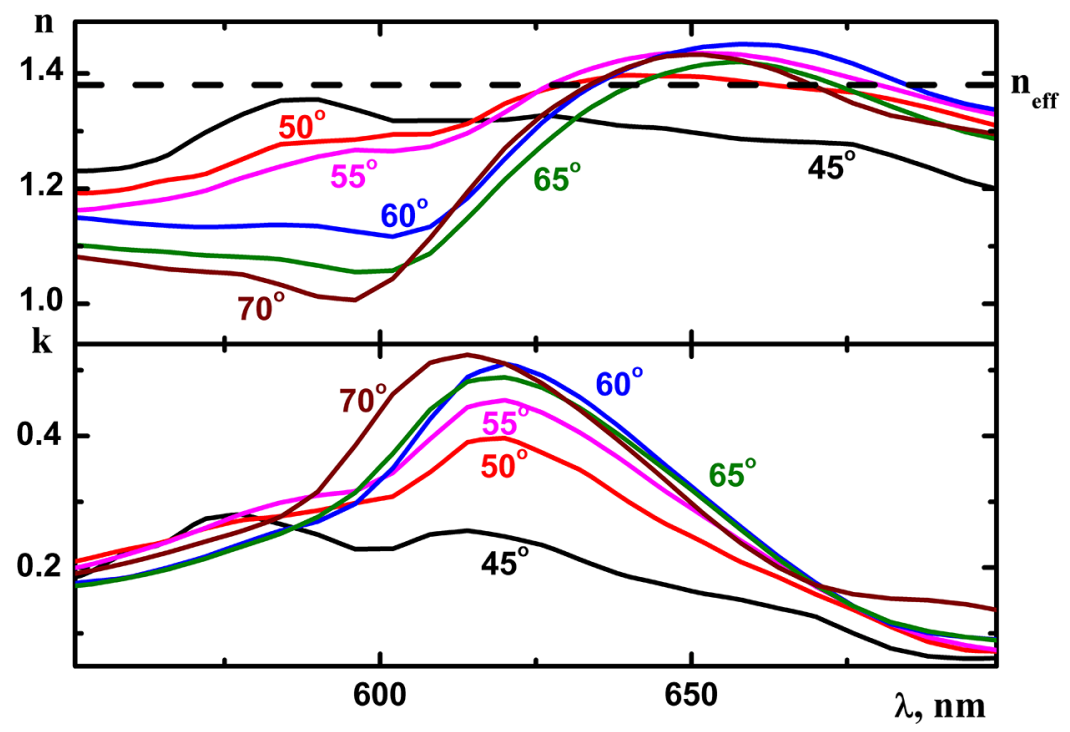

Figure 5 Refractive index $n(\lambda)$ and extinction coefficient $k(\lambda)$ of PMMA thin opal film calculated from ellipsometric data at different angles of light incidence. 


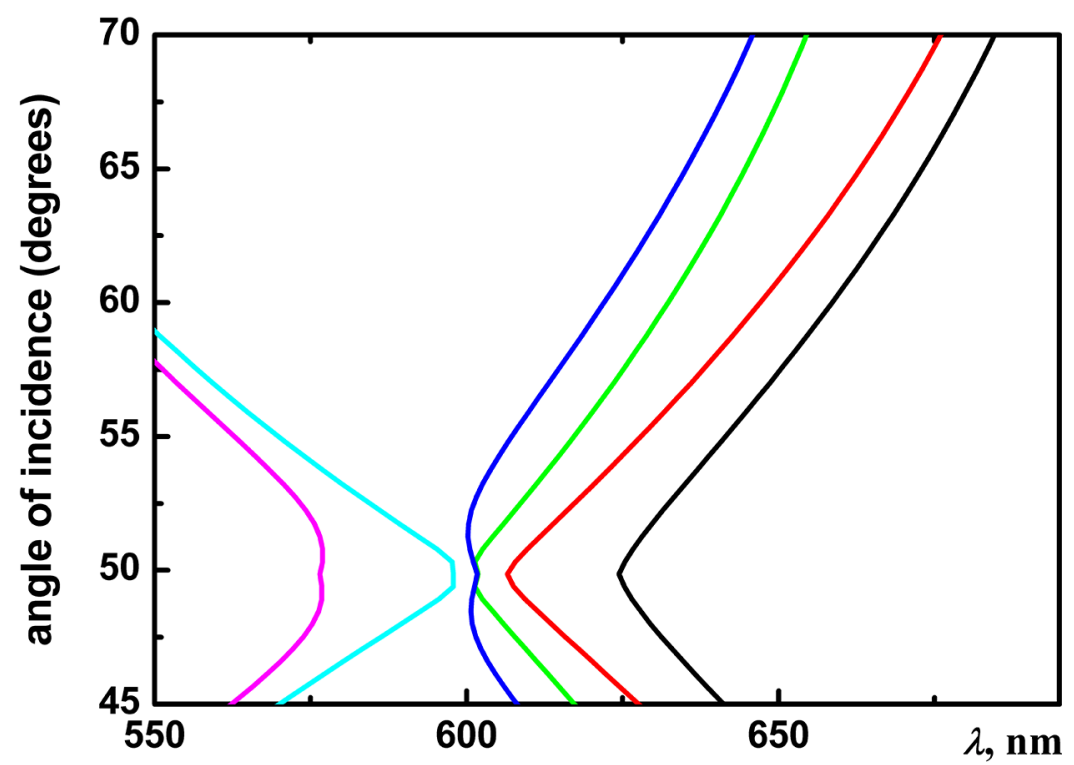

Figure 6 Fragment of the band structure of the opal film in the $\Gamma L K L$ cross-section of the FCC Brillouin zone.

the polarizability of the material, in PhCs this index depends also on the light interaction with the crystal structure, so that both factors affect the mode dispersion. As the result, the group velocity and the mode index are determined by the mode dispersion. Another specific feature of 3-dimensional $\mathrm{PhCs}$ is that the incident light excites eigenmodes through their projections on the surface of the Brillouin zone and different eigenmodes can be coupled simultaneously. In such circumstances, the index obtained from ellipsometry data can approach the value obtained from effective medium approximation, if and only if all excited eigenmodes possess linear dispersion with similar mode index. These conditions are nearly fulfilled in the long wavelength part of the studied spectral interval, where $n_{\text {eff } f}$ closely approximates $n(\lambda)$. Accordingly, at the opposite end of the spectral range the steeper dispersion of eigenmodes leads to lower value of $n(\lambda)$.

The interpretation of the imaginary part is more straightforward. The peak of light losses centered at $\sim 620 \mathrm{~nm}$ corresponds to the range of avoided band crossing, where different eigenmodes are strongly interacted each other. This interaction facilitates the efficient exchange of energy between different modes. As the result of such redistribution the light energy in the zero 


\section{Natalia Alekseeva et.al}

diffraction order is reduced. In turn, such reduction is counted by ellipsometric parameters as the increased magnitude of losses and produces a band in $k(\lambda)$ spectra. Owing to the multidirectional E-field distribution in Bloch modes such energy transfer becomes the angle-independent property.

\section{Conclusion}

We have shown that the ellipsometry provides complementary information to the data obtained by conventional optical characterization of opal crystals. This high-sensitive experimental technique makes it possible to discover some additional features in the wavelength- and angle-dependent optical response of these materials, especially at large angles of light incidence, near the Brewster angle $\theta_{B}\left(\tan \theta_{B}=n_{\text {eff }}\right)$ where the difference between s- and p- polarized electromagnetic waves becomes pronounced. The added value of the ellipsometry is that it can determine the effective index of refraction $n_{\text {eff }}$ without assuming any material parameters [7]. As it was shown in Section 3, the obtained index value corroborates the estimates based on the angular dispersion of Bragg resonance or on the effective medium approximation in the spectral range of the linear dispersion of opal eigenmodes.

The unique observation provided by ellipsometry is the detection of the energy exchange between $\mathrm{PhC}$ eigenmodes in the range of avoided band crossing, which appears in linear transmission and reflectance spectra as the doubling of the diffraction resonance.

\section{Acknowledgments}

The authors are grateful to Prof. M. I. Samoilovich for providing high-quality bulk opal matrices. This work was supported by the Ministry of Education and Science of Russian Federation according to the program "Development of Scientific Potential of Higher Educational Institutions", by German Academic Exchange Service (DAAD) and DFG funded Cluster of Excellence "Engineering of Advanced Materials" (Germany).

\section{References}

[1] V. N. Astratov, V. N. Bogomolov, A. A. Kaplyanskii, A. V. Prokofiev, L. A. Samoilovich, S. M. Samoilovich, Yu. A. Vlasov, Il Nuovo Cimento, 17D, 1349-1354 (1995). 
[2] S. G. Romanov, N. Gaponik, A. Eychmüller, A. L. Rogach, V. G. Solovyev, D. N. Chigrin, C. M. Sotomayor Torres, In Photonic crystals: Advances in design, fabrication, and characterization; Busch K.; Lölkes S.; Wehrspohn R. B.; Föll H.; Eds.; Weinheim, DE (2004).

[3] V. Solovyev, Y. Kumzerov, S. Khanin, Physics of regular matrix composites (Electrical and optical phenomena in nanocomposite materials based on porous dielectric matrices); Saarbrücken, DE, 2011 (in Russian).

[4] V. G. Balakirev, V. N. Bogomolov, V. V. Zhuravlev, Y. A. Kumzerov, V. P. Petranovskii, S. G. Romanov, L. A. Samoilovich, Crystallography Reports, 38, 348-353 (1993).

[5] V.A. Tkal, N. A. Voronin, V. G. Solov'ev, N. O. Alekseeva, S. V.Pan'kova, and M. V. Yanikov, Inorganic Materials, 46, 119-121 (2010).

[6] V. A. Shvets, E. V. Spesivtsev, S. V. Rykhlitskii, and N. N. Mikhailov, Nanotechnologies in Russia, 4, 201-214 (2009).

[7] M. Ahles, T. Ruhl, G. P. Hellmann, H. Winkler, R. Schmechel, H. von Seggern, Optics Communications, 246, 1-7 (2005).

[8] A. I. Plekhanov, V. P. Chubakov, and P. A. Chubakov, Physics of the Solid State, 53, 1145-1151 (2011).

[9] A. Reza, Z. Balevicius, R. Vaisnoras, G. J. Babonas, A. Ramanavicius, Thin Solid Films, 519, 2641-2644 (2011).

[10] W. Khunsin, A. Amann, G. Kocher, S. G. Romanov, S. Pullteap, H. C. Seat, E. P. O'Reilly, R. Zentel, C. M. Sotomayor Torres, Adv. Func. Mater., 22, 1812-1821 (2012).

[11] A. F. Belyanin, M. I. Samoilovich, Nanostructures and Photon Crystals: Collective Monograph after the Materials of Plenary Reports of the $10^{\text {th }}$ International Conference "High Technology in Russian Industry"; Moscow: CRTI "Technomash" (2004).

[12] S. G. Romanov, T. Maka, C. M. Sotomayor Torres, M. Müller, R. Zentel, D. Cassagne, J. Manzanares-Martinez, and C. Jouanin, Phys. Rev. E, 63, 056603 (2001).

[13] V. G. Solovyev, S. G. Romanov, D. N. Chigrin, C. M. Sotomayor Torres, Synthetic Metals, 139, 601 (2003).

[14] N. Alekseeva, V. Veisman, A. Lukin, S. Pan'kova, V. Solovyev, M. Yanikov, Nanotechnics, 31, 23-26 (2012) (in Russian).

[15] C. Wolff, S. G. Romanov, J. Küchenmeister, U. Peschel and K. Busch, submitted. 


\section{Natalia Alekseeva et.al}

\section{Biographies}

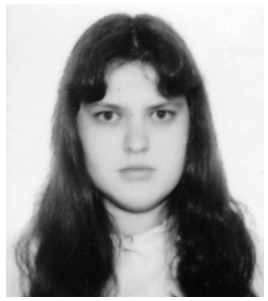

Natalia Alekseeva finished her post-graduate course in Solid State Physics at Pskov State Pedagogical University (Russia) in 2010. Her research activity is mostly in the fields of the scanning probe microscopy (SPM) and physics of nanostructures based on porous alumina.

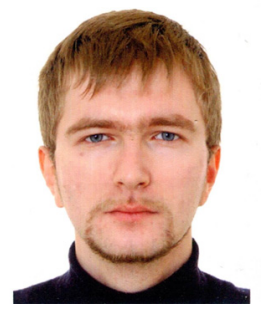

Grigory Cema is a third-year post-graduate student in Physics of Condensed Matter at Pskov State University (Russia). His research is focused on the study of optical characteristics of nanocomposite materials by photoluminescence and ellipsometry as well as on X-ray physics.

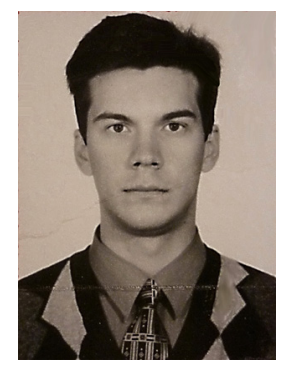

Aleksey Lukin is a third-year post-graduate student in Solid State Physics at Pskov State University (Russia). His research is focused on the electrical 
characterisation of zeolite-based nanocomposites as well as on the preparation of opal-based metal-dielectric systems.

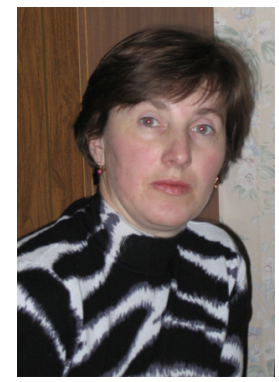

Svetlana Pan'kova graduated in Physics and Mathematics in 1987 and got her Ph.D. in Solid State Physics at Herzen State Pedagogical University of Russia (St. Petersburg) in 1998. Today Svetlana Pan'kova is Associate Professor at Pskov State University. Her research activity is mostly in the fields of the scanning probe microscopy and electrical characterisation of opal-based nanostructures.

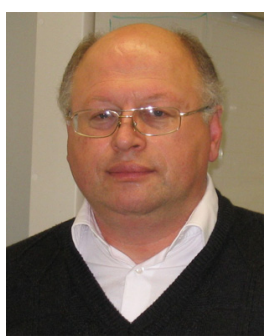

Sergei Romanov received his Diploma from the Polytechnical Institute of Leningrad, USSR in 1978. He obtained PhD and DSc degrees from the Ioffe Institute in 1986 and 2013. Since 1990 he is a research professor at Ioffe Institute. He authored a number of pioneering results in the physics of regular ensembles of templated nanostructures. He also promoted this approach to realization of low-dimensional materials working at universities in Glasgow, Wuppertal, Cork and Erlangen. He currently focused on designing complex photonic and plasmonic architectures based on colloidal platforms. 


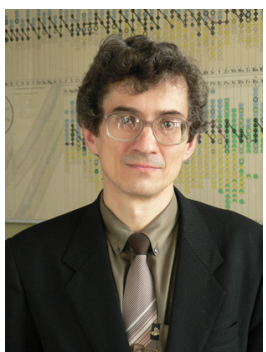

Vladimir Solovyev got his diploma from Pskov State Pedagogical Institute (USSR) in 1976, his PhD and DSc degrees from Herzen State Pedagogical University of Russia (St. Petersburg) in 1991 and 2005. Today he is a Professor, Head of Physics Department at Pskov State University. He is the author of about 50 regular papers and 3 monographs. Major research interests focus around electrical and optical phenomena in nanocomposite materials based on regular porous dielectric matrices.

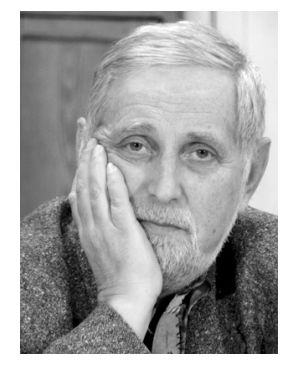

Victor Veisman got his diploma from Pskov State Pedagogical Institute (USSR). Today he is Associate Professor at Pskov State University. He is the author of more than 100 regular papers, supervisor of dozens Diploma students, experienced lecturer on optics, atomic and solid state physics, quantum mechanics and astronomy. His research interests are in electrical and optical properties of point defects in alkali halide crystals, as well as in methods of preparation of nanocomposites based on regular porous dielectric matrices. 


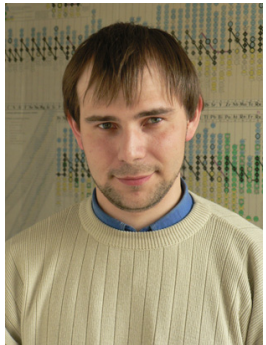

Mikhail Yanikov finished his post-graduate course in Solid State Physics at Pskov State Pedagogical University (Russia) in 2008. His research activity is mostly in the field of reflectance and transmission angle-resolved optical spectroscopy of opal-based 3-dimensional photonic crystals. 
